\title{
Non-invasive High-Frequency Oscillatory Ventilation as Initial Respiratory Support for Preterm Infants With Respiratory Distress Syndrome
}

\begin{abstract}
Shu-Hua Lai 1,2, Ying-Ling Xie ${ }^{1,2}$, Zhi-Qing Chen ${ }^{1,2}$, Rong Chen ${ }^{1,2}$, Wen-Hong Cai ${ }^{1,2}$, Luo-Cheng $W_{u^{1,2}}$, Yun-Feng Lin ${ }^{1,2,3,4 *}$ and Yi-Rong Zheng ${ }^{1,2,3,4 *}$

${ }^{1}$ Department of Neonatology, Fujian Maternity and Child Health Hospital, Affiliated Hospital of Fujian Medical University, Fuzhou, China, ${ }^{2}$ Fujian Key Laboratory of Women and Children's Critical Diseases Research, Fujian Maternity and Child Health Hospital, Fuzhou, China, ${ }^{3}$ Fujian Branch of Shanghai Children's Medical Center, Fuzhou, China, ${ }^{4}$ Fujian Children's Hospital, Fuzhou, China
\end{abstract}

Objectives: The aim of this study was to investigate the safety and feasibility of nHFOV as initial respiratory support in preterm infants with RDS.

Edited by: Deb Strickland,

University of Western Australia, Australia

Reviewed by:

Daniel Klotz,

University of Freiburg, Germany

Gianluca Lista,

Ospedale dei Bambini Vittore

Buzzi, Italy

${ }^{*}$ Correspondence:

Yun-Feng Lin

linyf2003@qq.com

Yi-Rong Zheng

zhengyirong2020@163.com

Specialty section: This article was submitted to

Neonatology,

a section of the journa

Frontiers in Pediatrics

Received: 09 October 2021 Accepted: 17 December 2021

Published: 11 January 2022

Citation:

Lai SH, Xie YL, Chen ZQ, Chen R, Cai WH, Wu LC, Lin YF and Zheng YR

(2022) Non-invasive High-Frequency Oscillatory Ventilation as Initial Respiratory Support for Preterm Infants With Respiratory Distress

Syndrome. Front. Pediatr. 9:792160.

doi: $10.3389 /$ fped.2021.792160
Methods: This study retrospectively analyzed the clinical data of 244 premature infants with RDS who were treated in our hospital from January 2016 to January 2019 and divided into the nHFOV group $(n=115)$ and the BiPAP group $(n=129)$ based on the initial respiratory support method.

Results: Respiratory outcomes showed that the rate of NIV failure during the first 72 hours of life in the nHFOV group was significantly lower than that in the BiPAP group. The time of NIV in the nHFOV group was significantly shorter than that in the BiPAP group. The time of supplemental oxygen in the nHFOV group was significantly shorter than that in the BiPAP group. The incidence of air leakage syndrome in the nHFOV group was significantly lower than that in the BiPAP group, and the length of hospital stay of the $\mathrm{nHFOV}$ group was also significantly shorter than that in the BiPAP group. Although the rate of infants diagnosed with BPD was similar between the two groups, the rate of severe BPD in the nHFOV group was significantly lower than that in the BiPAP group.

Conclusion: This study showed that nHFOV as initial respiratory support for preterm infants with RDS was feasible and safe compared to BiPAP. Furthermore, nHFOV can reduce the need for IMV and reduce the incidence of severe BPD and air leak syndrome.

Keywords: non-invasive high-frequency oscillatory ventilation, biphasic positive airway pressure, preterm infants, respiratory distress syndrome, non-invasive ventilation

\section{INTRODUCTION}

Respiratory distress syndrome (RDS) is one of the most common complications in preterm infants and the most common reason for premature death. A large proportion of preterm infants with RDS require invasive mechanical ventilation (IMV) at an early stage of life. Although ventilation is usually life-saving, it can also cause many complications, such as air leak syndrome, lung injury, 
and neurodevelopmental impairment (1-3). Neonatologists are increasingly using non-invasive ventilation (NIV) in the neonatal intensive care unit (NICU) to reduce these adverse effects of IMV; among them, biphasic positive airway pressure (BiPAP) is the classic non-invasive ventilation mode. Reports have shown that NIV is feasible in clinical practice and is associated with reducing the need for intubation and decreasing ventilatorrelated lung injury and other complications $(2,4)$. Non-invasive high-frequency oscillatory ventilation (nHFOV) is a promising new mode of NIV that can reduce the risk and complications of $\operatorname{IMV}(5,6)$. However, there are few reports on the application of nHFOV for the treatment of premature infants with RDS. We hypothesized that nHFOV was safe and effective as an initial respiratory support for preterm infants with RDS and had more advantages than BiPAP. We conducted a retrospective controlled study to evaluate the efficacy, safety and advantages of nHFOV for the treatment of premature infants with RDS.

\section{METHODS}

The present study was approved by the ethics committee of our hospital and adhered to the tenets of the Declaration of Helsinki. Additionally, all parents of the patients signed the consent form before participating in the study.

\section{Patients}

Our hospital started using nHFOV in January 2018, and then RDS initial respiratory support was gradually transitioned from the previous BiPAP to $\mathrm{nHFOV}$. Therefore, BiPAP respiratory support was also used in some patients from 2018 and 2019. From January 2016 to January 2019, there was no change in other treatment except respiratory support. This study retrospectively analyzed the clinical data of 244 premature infants with RDS who were treated in our hospital from January 2016 to January 2019 and were divided into two groups based on the initial respiratory support methods. The nHFOV group had 115 premature infants who received nHFOV as initial respiratory support, and the BiPAP group had 129 premature infants who received BiPAP as initial respiratory support (Figure 1). All the patients were definitely diagnosed with RDS based on the diagnostic criteria. The diagnostic criteria of RDS are as follows (6): (1) Highrisk factors: maternal diabetes during pregnancy, intrauterine infection, premature delivery, premature rupture of membranes for more than $24 \mathrm{~h}$, intrauterine distress, asphyxia during delivery, etc. (2) Clinical symptoms: progressive aggravation of tachypnea within $6 \mathrm{~h}$ after birth (>60 times/min); cyanosis, three depressions in inhalation and obvious expiratory moans, irregular breathing, and apnoea; and decreased respiratory

Abbreviations: RDS, Respiratory distress syndrome; IMV, Invasive mechanical ventilation; NIV, Non-invasive ventilation; NICU, Neonatal intensive care unit; BiPAP, Biphasic positive airway pressure; nHFOV, Non-invasive highfrequency oscillatory ventilation; FiO2, Inspired oxygen; MAP, Average airway pressure; NCPAP, Nasal continuous positive airway pressure; BPD, Bronchopulmonary dysplasia. sounds in both lungs were detected on auscultation. (3) Typical chest X-ray features.

The inclusion criteria were as follows: (1) premature infants with gestational age of 25-34 weeks; (2) diagnosed with RDS within $24 \mathrm{~h}$ of birth and received nHFVO or BiPAP as initial respiratory support. The exclusion criteria were as follows: (1) complications involving severe congenital structural malformations such as congenital heart disease, congenital diaphragmatic hernia, respiratory tract malformation, and severe digestive tract malformation; (2) incomplete data; and (3) the parents of the infants refused to participate in this study.

\section{Management in the Delivery Room}

Drying, maintaining warmth and treating the umbilical cord immediately after birth were performed in the delivery room. Then, based on the breathing condition of the premature baby, we started respiratory support in the delivery room with nasal continuous positive airway pressure support or intermittent positive pressure ventilation after intubation. The initial management in the delivery room for all the babies in the two groups was the same.

\section{Respiratory Management}

Daily care was performed as needed for preterm infants in the NICU, along with continuous monitoring of pulse oxygen saturation, respiratory rate, and heart rate. $\mathrm{nHFOV}$ or BiPAP was started within $24 \mathrm{~h}$ of life with clinical signs of respiratory distress. Short binasal prongs (Infant Flow, CareFusion, California, USA) were used as the interface for the two NIV devices. The orogastric tube was kept open to decompress the stomach and to facilitate feeding. All of the preterm infants were given prophylactic caffeine on the first day of life. Caffeine was administered at a loading dose of $20 \mathrm{mg} / \mathrm{kg}$ caffeine citrate, and then a daily maintenance dose of $5-10 \mathrm{mg} / \mathrm{kg}$ coffee citrate was given. Our unit did not implement preventive use of surfactants. Surfactant requirements were assessed for all preterm infants. Porcine surfactant (Curosurf, Chiesi Farmaceutici, Parma, Italy) was administered as a rescue therapy via the intubation, surfactant therapy, extubation (INSURE) method if the infant required $\geq$ 0.40 fraction of inspired oxygen $(\mathrm{FiO} 2)$ to maintain the target oxygen saturation level of $90-95 \%$. The first dose of surfactant therapy was $200 \mathrm{mg} / \mathrm{kg}$. Additional surfactant doses of $100 \mathrm{mg} / \mathrm{kg}$ were administered if the infants still required $\geq 0.40 \mathrm{FiO} 2$ to maintain the target oxygen saturation.

In the nHFOV group, a neonatal non-invasive high-frequency ventilator from Medin $\mathrm{CNO}$ (Medical Innovations $\mathrm{GmbH}$, Puchheim, Germany) with an output oxygen concentration range of 21 to $100 \%$ was used. The initial parameters were as follows: average airway pressure (MAP) was $8 \mathrm{cmH}_{2} \mathrm{O}$, frequency was $9 \mathrm{~Hz}$, amplitude was adjusted to achieve sufficient chest oscillation at rest, and $\mathrm{FiO} 2$ was 0.4 . Based on blood gas analysis and transcutaneous oxygen saturation ( $\mathrm{SpO} 2)$ adjustment parameters, FiO2 was adjusted by 0.05 , MAP was adjusted by $1 \mathrm{cmH}_{2} \mathrm{O}$, and frequency was adjusted by $1 \mathrm{~Hz}$ each time.

For infants in the BiPAP group, a baseline PEEP of 5 $\mathrm{cmH}_{2} \mathrm{O}$ was established, with a high PEEP of $9 \mathrm{cmH}_{2} \mathrm{O}$, a 


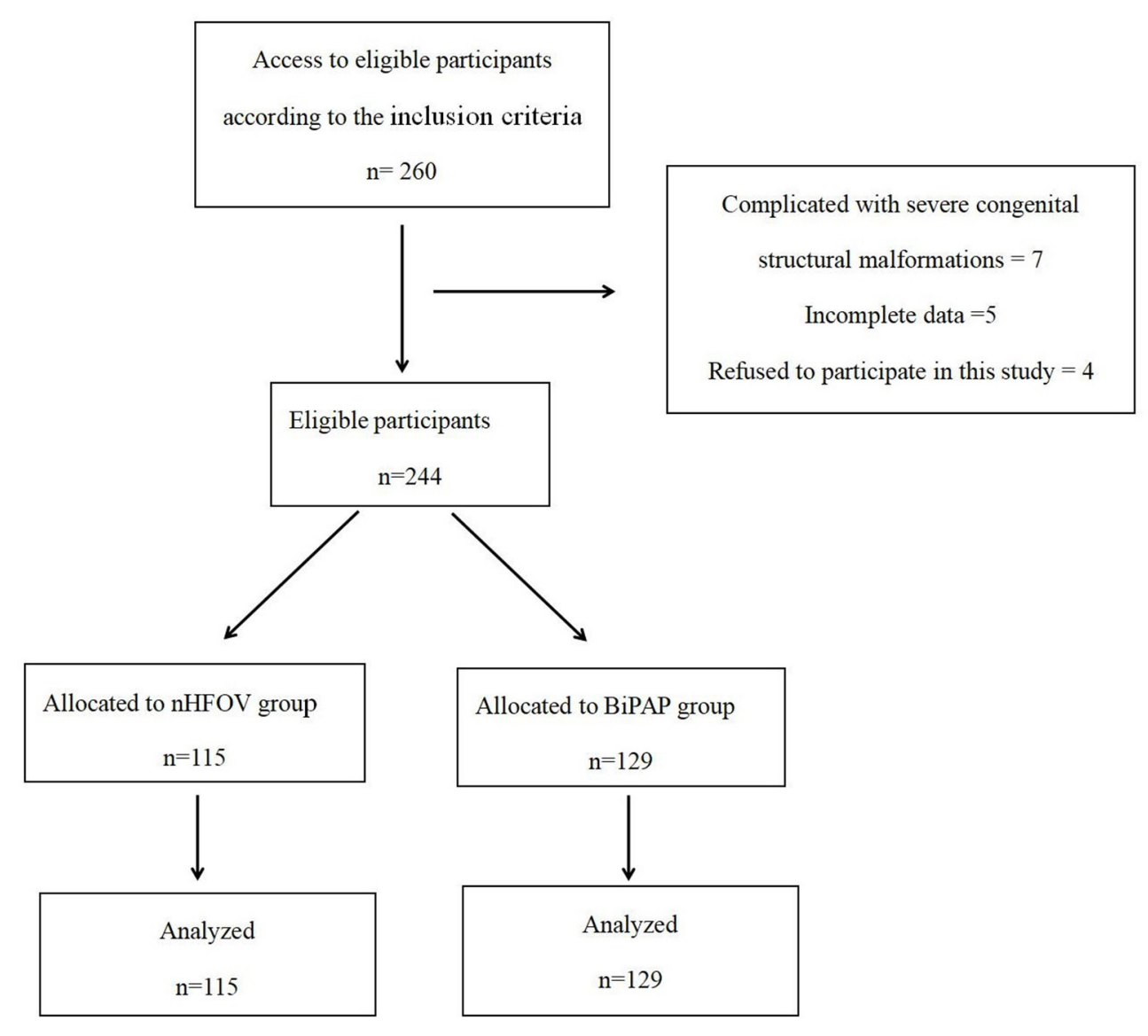

FIGURE 1 | The patient flow diagram of the study.

respiratory rate of $30 / \mathrm{min}$, and an initial inspiratory time of $0.5 \mathrm{~s}$ (CareFusion, California, USA). The baseline PEEP was adjusted to $4-7 \mathrm{cmH}_{2} \mathrm{O}$, and the high PEEP was adjusted to $7-10 \mathrm{cmH}_{2} \mathrm{O}$.

If the infant remained clinically stable at minimum respiratory parameters with nHFOV (MAP: $6 \mathrm{cmH} 2 \mathrm{O}$; FiO2: 0.30) and BiPAP (cycle rate: 15 times/min, lower CPAP: $3 \mathrm{cmH}_{2} \mathrm{O}$; higher CPAP: $5 \mathrm{cmH}_{2} \mathrm{O} ; \mathrm{FiO} 2: 0.30$ ), good respiratory effort and maintenance of an oxygen saturation level of 90-95\%, conditions were switched to a heated humidified high-flow nasal cannula or nasal continuous positive airway pressure (NCPAP). If the infant under NCPAP had a CPAP level of $3 \mathrm{cmH}_{2} \mathrm{O}$ and $\mathrm{FiO} 2<0.25$ and tolerated the treatment well for at least $24 \mathrm{~h}$ with no evidence of apnoea, the infant was weaned to supplemental oxygen or room air.

Failure of NIV was defined as at least one of the following (7): severe respiratory acidosis ( $\mathrm{pH} \leq 7.20$ and $\mathrm{PaCO} 2 \geq 60 \mathrm{mmHg})$; hypoxemia $\left(\mathrm{PaO}_{2} \leq 50 \mathrm{mmHg}\right.$ and $\mathrm{FiO} 2 \geq 0.6$ ); recurrent apnoea associated with bradycardia $\geq 3$ times/hour; or a single episode of apnoea that required bag-and-mask ventilation, pneumothorax or intestinal perforation, severe respiratory distress, or pulmonary hemorrhage.
The definition of bronchopulmonary dysplasia (BPD) was any oxygen dependence $\left(\mathrm{FiO}_{2}>21 \%\right)$ of the newborn lasting more than 28 days. Based on the judgement of oxygen dependence and different types of respiratory support measures, BPD can be categorized into three degrees: mild, moderate and severe. Preterm infants with a gestational age less than 32 weeks were assessed at 36 weeks postmenstrual or discharge, and preterm infants with a gestational age more than 32 weeks were assessed at 56 days after birth or discharge. BPD was considered (1) mild for patients without oxygen inhalation; (2) moderate for those requiring oxygen inhalation and $\mathrm{FiO} 2$ was < 30\%; and (3) severe for those requiring oxygen inhalation, $\mathrm{FiO} 2$ was $\geq 30 \%$ or positive pressure ventilation was needed (8).

\section{Statistical Analysis}

SPSS 25.0 was used for statistical analysis. Continuous data are presented as the mean \pm standard deviation and range. With all continuous data, the normality of the distribution was tested, and they followed a normal distribution. Clinical parameters between the two groups were compared with independent samples $t$ tests. $\chi 2$ tests were used to categorize the variables. A $p$ value of $<0.05$ was defined as significant. 
TABLE 1 | Comparison of general data between the two groups.

\begin{tabular}{|c|c|c|c|}
\hline & nHFOV group & BiPAP group & $P$-value \\
\hline Number & 115 & 129 & \\
\hline Gestational age (weeks) & $29.1 \pm 1.8$ & $28.9 \pm 1.6$ & 0.457 \\
\hline Birthweight (kg) & $1.1 \pm 0.220$ & $1.2 \pm 0.242$ & 0.198 \\
\hline Male/female & $59 / 56$ & $68 / 61$ & 0.826 \\
\hline Multiple births & $14(12.1 \%)$ & $17(13.2 \%)$ & 0.814 \\
\hline Apgar score at 5 min & $8.2 \pm 2.1$ & $8.3 \pm 2.0$ & 0.979 \\
\hline Intra uterine growth retardation & $21(18.3 \%)$ & $26(20.2 \%)$ & 0.708 \\
\hline Ante-natal steroids (incomplete and full course) & $61(53.0 \%)$ & $78(60.4 \%)$ & 0.242 \\
\hline Cesarean section & $55(47.8 \%)$ & $74(57.4 \%)$ & 0.136 \\
\hline Gestational diabetes mellitus & $35(30.4 \%)$ & $46(35.7 \%)$ & 0.387 \\
\hline Pre-eclampsia & 24 (20.9\%) & $29(22.5 \%)$ & 0.763 \\
\hline Maternal age (years) & $29.2 \pm 3.0$ & $29.3 \pm 2.5$ & 0.744 \\
\hline Prolonged premature rupture of membranes $>18 \mathrm{~h}$ & $26(22.6 \%)$ & $22(17.1 \%)$ & 0.276 \\
\hline Chorioamnionitis & $13(11.3 \%)$ & $10(7.8 \%)$ & 0.343 \\
\hline
\end{tabular}

BiPAP, biphasic positive airway pressure; nHFOV, non-invasive high-frequency oscillatory ventilation.

\section{RESULTS}

The general data, birth situation and perinatal data of the participants in the two groups are shown in Table 1; there were no statistically significant differences between the two groups, which indicated that the baseline characteristics between the two groups were similar (Table 1).

The respiratory outcomes showed that the rate of NIV failure in the first $72 \mathrm{~h}$ of life was significantly lower in the nHFOV group than in the BiPAP group ( 9 vs. $23, P=0.021$ ). The time of NIV in the nHFOV group was shorter than that in the BiPAP group (10.5 \pm 4.3 vs. $12.7 \pm 5.6, P=0.032$ ). Among infants with NIV failure, the time of first intubation in the BiPAP group was significantly earlier than that in the nHFOV group ( $2.0 \pm 1.6$ vs. $3.5 \pm 2.3, P=$ 0.048 ), and the time of invasive ventilation in the nHFOV group was significantly shorter than that in the BiPAP group ( $3.2 \pm 1.8$ vs. $5.4 \pm 2.0, P=0.036$ ). The time of supplemental oxygen in the nHFOV group was shorter than that in the BiPAP group (7.2 \pm 4.8 vs. $8.5 \pm 5.6, P=0.040$ ). The rate of the required surfactant was similar between the two groups (Table 2).

A comparison of the complications between the two groups showed that the incidence of air leakage syndrome in the nHFOV group was significantly lower than that in the BiPAP group (2 vs. $10, P=0.038$ ), and the length of hospital stay was also significantly shorter in the nHFOV group than in the BiPAP group ( $44.0 \pm 10.9$ vs. $47.3 \pm 12.8, P=0.033)$. Although the rate of infants diagnosed with BPD was similar between the two groups, the rate of severe BPD in the nHFOV group was significantly lower than that in the BiPAP group ( 1 vs. $7, P=$ 0.046 ). The incidence of nasal injury, retinopathy of prematurity requiring laser treatment, necrotizing enterocolitis, and mortality were similar between the two groups (Table 3).

\section{DISCUSSION}

Various forms of NIV are increasingly being used by neonatologists because of the potential adverse consequences of IMV, particularly ventilator-induced lung injury, and this restricted use of IMV in preterm infants might decrease lung inflammation and reduce the incidence of BPD and death (9). The most commonly used NIV method is BiPAP, and BiPAP is an effective therapy in the early management of RDS in preterm infants (10). nHFOV, as a novel mode of non-invasive ventilation, can improve the removal of carbon dioxide with the advantages of high-frequency ventilation and NIV (11).

Studies have shown that nHFOV can reduce the need for IMV compared to other NIV techniques (12-14). In this study, we found that compared to BiPAP, nHFOV as initial respiratory support reduced the need for IMV within the first $72 \mathrm{~h}$ of life. We also found that nHFOV decreased the rate of intubation, shortened the duration of IMV and postponed the first intubation. Compared with other forms of NIV, nHFOV retains a non-invasive interface and maintains continuous airway pressure, and it can also increase functional residual volume, maintain the opening of the upper airway, and prevent alveolar collapse, which can improve ventilation and oxygenation (15). Mukerji et al. used a lung model to compare nHFOV with other forms of NIV, and the results showed that nHFOV could remove carbon dioxide more effectively, promote alveolar revascularization and reduce the rate of tracheal intubation compared with other forms of NIV (16). We suggested that the higher NIV failure rate in the BiPAP group than in the nHFOV group might also be due to BiPAP offering synchronized nasal intermittent positive pressure ventilation using an abdominal capsule, which may be difficult to synchronize with infant breathing and does not result in larger tidal volumes (17). However, with nHFOV, there is no need for synchronization. nHFOV may improve ventilation by enhancing alveolar recruitment by applying higher MAP, and the functional residual capacity was increased (18). On the other hand, despite restricting the use of MAP on nHFOV to $10 \mathrm{cmH}_{2} \mathrm{O}$, this was still higher than BiPAP for the corresponding $\mathrm{FiO}_{2}$ levels. Binmanee et al. (19) proved that the use of high NIV (MAP $\left.\geq 10 \mathrm{cmH}_{2} \mathrm{O}\right)$ resulted in the avoidance of intubation in the majority of cases, 
TABLE 2 | Comparison of respiratory status between the two groups.

\begin{tabular}{|c|c|c|c|}
\hline & nHFOV group & BiPAP group & $P$-value \\
\hline Number & 115 & 129 & \\
\hline NIV failure in the first 72 hours of life & $9(7.8 \%)$ & $23(17.8 \%)$ & 0.021 \\
\hline Required surfactant & $46(40.0 \%)$ & $59(45.7 \%)$ & 0.366 \\
\hline Required $\geq 2$ doses of surfactant & $10(8.7 \%)$ & $17(13.1 \%)$ & 0.265 \\
\hline Time at first intubation (days) & $3.5 \pm 2.3$ & $2.0 \pm 1.6$ & 0.048 \\
\hline Duration of NIV (days) & $10.5 \pm 4.3$ & $12.7 \pm 5.6$ & 0.032 \\
\hline Duration of IMV (days) & $3.2 \pm 1.8$ & $5.4 \pm 2.0$ & 0.036 \\
\hline Duration of supplemental oxygen (days) & $7.2 \pm 4.8$ & $8.5 \pm 5.6$ & 0.040 \\
\hline
\end{tabular}

BiPAP, Biphasic positive airway pressure; nHFOV, non-invasive high-frequency oscillatory ventilation; IMV, invasive mechanical ventilation; NIV, Non-invasive ventilation.

TABLE 3 | Comparison of complications between the two groups.

\begin{tabular}{|c|c|c|c|}
\hline & nHFOV group & BiPAP group & $P$-value \\
\hline Number & 115 & 129 & \\
\hline Air leak syndrome & $2(1.7 \%)$ & $10(7.8 \%)$ & 0.038 \\
\hline Retinopathy of prematurity required laser treatment & $4(3.5 \%)$ & $9(7.0 \%)$ & 0.225 \\
\hline Necrotizing enterocolitis & $8(7.0 \%)$ & $15(11.6 \%)$ & 0.213 \\
\hline Moderate BPD & $6(5.2 \%)$ & $9(7.0 \%)$ & 0.568 \\
\hline Severe BPD & $1(0.9 \%)$ & $7(5.4 \%)$ & 0.046 \\
\hline Duration of hospitalization (days) & $44.0 \pm 10.9$ & $47.3 \pm 12.8$ & 0.033 \\
\hline Mortality & $7(6.1 \%)$ & $8(6.2 \%)$ & 0.970 \\
\hline
\end{tabular}

BiPAP, biphasic positive airway pressure; $\mathrm{HHFOV}$, non-invasive high-frequency oscillatory ventilation; BPD, bronchopulmonary dysplasia.

without adverse effects. Yaser et al. (20) also found a higher MAP in the successful group than in the failure group as a prophylactic or rescue mode of NIV following extubation. Therefore, these differences may be due to the airway pressure itself rather than the pressure waveform generated from the two NIV modes.

The nHFOV group had a lower rate of air leak syndrome than the BiPAP group, and we suspected that this may be due to the small tidal volumes used, which results in less pressure-andvolume trauma from nHFOV (21). High-frequency oscillation ventilation is a type of oscillation with a high frequency of air flow. Through a diffusion mechanism, a small amount of gas was sent into or out of the airway ventilation method, and there was no synchronization and no man-machine confrontation (22). However, BiPAP offers synchronized nasal intermittent positive pressure ventilation using an abdominal capsule, which may be difficult to synchronize with infant breathing. This situation makes it very easy to create man-machine confrontation; even if the MAP was lower, air leak syndrome was more likely to occur.

$\mathrm{BPD}$ is a common and serious complication in premature infants (23). Studies have shown that IMV and high oxygen exposure are high-risk factors for $\operatorname{BPD}(24,25)$. Although the rate of infants diagnosed with BPD was similar between the two groups, the rate of severe BPD in the nHFOV group was significantly lower than that in the BiPAP group. This difference may have resulted from the facilitation of gas exchange in neonates treated with nHFOV, and nHFOV was able to sustain oxygenation and ventilation while leading to improved alveolar or lung development $(6,26-28)$. These effects on lung development raise the possibility that nHFOV may reduce neonatal chronic lung disease. nHFOV reduces the duration of supplemental oxygen and the use of IMV, which can also help reduce the incidence of severe $\mathrm{BPD}$.

nHFOV reduced the duration of supplemental oxygen and hospitalization, which may have benefitted from the reduction in airway inflammation, lung injury, and incidence of severe BPD by $n \mathrm{HFOV}$. Of concern was the decrease in NEC and retinopathy of prematurity requiring laser treatment in the nHFOV group, which may have been due to nHFOV reducing the duration of supplemental oxygen and IMV and the fluctuations in blood oxygen saturation, although there were no statistically significant differences.

Our study had some limitations, which may affect the validity of our findings. First, it was a retrospective study, and it was not a prospective randomized controlled study, and therefore, the study's objectivity was somewhat limited. Second, it was a single-center study with a small sample size. Third, the follow-up time was not long enough. Fourth, the study infants were not stratified by birth weight or gestational 
age, and there was a lack of infants at less than 25-weeks of gestation.

\section{CONCLUSION}

This study showed that nHFOV as initial respiratory support in preterm infants with RDS was feasible and safe compared to BiPAP. Furthermore, nHFOV can reduce the need for IMV and reduce the incidence of severe BPD and air leak syndrome.

\section{DATA AVAILABILITY STATEMENT}

The data analyzed in this study is subject to the following licenses/restrictions: The data that support the findings of this study are available on request from the corresponding author.

\section{REFERENCES}

1. Miller JD, Carlo WA. Pulmonary complications of mechanical ventilation in neonates. Clin Perinatol. (2008) 35:273-81. doi: 10.1016/j.clp.2007.11.004

2. Sweet DG, Carnielli V, Greisen G, Hallman M, Ozek E, Te Pas $\mathrm{A}$, et al. European consensus guidelines on the management of respiratory distress syndrome - 2019 update. Neonatology. (2019) 115:432-50. doi: 10.1159/000499361

3. Walsh MC, Morris BH, Wrage LA, Vohr BR, Poole WK, Tyson JE, et al. Extremely low birth weight neonates with protracted ventilation: mortality and 18-month neurodevelopmental outcomes. J Pediatr. (2005) 146:798804. doi: 10.1016/j.jpeds.2005.01.047

4. Vendettuoli V, Bellu R, Zanini R, Mosca F, Gagliardi L. Italian Neonatal Network: Changes in ventilator strategies and outcomes in preterm infants. Arch Dis Child Fetal Neonatal Ed. (2014) 99:F321-4. doi: 10.1136/archdischild-2013-305165

5. Zhu XW, Zhao JN, Tang SF, Yan J, Shi Y. Noninvasive highfrequency oscillatory ventilation versus nasal continuous positive airway pressure in preterm infants with moderate-severe respiratory distress syndrome: a preliminary report. Pediatr Pulmonol. (2017) 52:1038-42. doi: 10.1002/ppul.23755

6. Null DM, Alvord J, Leavitt W, Wint A, Dahl MJ, Presson AP, et al. Highfrequency nasal ventilation for $21 \mathrm{~d}$ maintains gas exchange with lower respiratory pressures and promotes alveolarization in preterm lambs. Pediatr Res. (2014) 75:507-16. doi: 10.1038/pr.2013.254

7. Shi Y, De Luca D. Continuous positive airway pressure (CPAP) vs noninvasive positive pressure ventilation (NIPPV) vs noninvasive high frequency oscillation ventilation (NHFOV) as post-extubation support in preterm neonates: protocol for an assessor-blinded, multicenter, randomized controlled trial. BMC Pediatr. (2019) 19:256. doi: 10.1186/s12887-0191625-1

8. Jobe AH, Bancalari E. Bronchopulmonary dysplasia. Am J Respir Crit Care Med. (2001) 163:1723-9. doi: 10.1164/ajrccm.163.7.2011060

9. Vliegenthart RJS, Onland W, van Wassenaer-Leemhuis AG et al. Restricted ventilation associated with reduced neurodevelopmental impairment in preterm infants. Neonatology. (2017) 112:172-9. doi: 10.1159/000471841

10. Buyuktiryaki M, Okur N, Sari FN, Bekmez BO, Bezirganoglu H, Cakir U, et al. Comparison of three different noninvasive ventilation strategies as initial respiratory support in very low birth weight infants with respiratory distress syndrome: a retrospective study. Arch Pediatr. (2020) 27:3227. doi: 10.1016/j.arcped.2020.06.002

11. Bottino R, Pontiggia F, Ricci C, Gambacorta A, Paladini A, Chijenas V, et al. Nasal high-frequency oscillatory ventilation and $\mathrm{CO} 2$ removal: a randomized controlled crossover trial. Pediatr Pulmonol. (2018) 53:124551. doi: $10.1002 /$ ppul. 24120
The data are not publicly available due to privacy or ethical restrictions. Requests to access these datasets should be directed to Shu-Hua Lai, laishuhua2014@163.com.

\section{AUTHOR CONTRIBUTIONS}

S-HL, Y-FL, and Y-RZ designed the study, collected the clinical data, performed the statistical analysis, participated in the operation, and drafted the manuscript. Y-LX, Z-QC, RC, W-HC, and L-CW participated in the operation and revised the article. All authors read and approved the final manuscript.

\section{ACKNOWLEDGMENTS}

We appreciated all doctors in our center for fruitful advice and discussions.
12. Mukerji A, Singh B, Helou SE, Fusch C, Dunn M, Belik J. et al. Use of noninvasive high-frequency ventilation in the neonatal intensive care unit: a retrospective review. Am J Perinatol. (2015) 30:171-6. doi: 10.1055/s-0034-1381317

13. Ali YAH, Seshia MM, Ali E, Alvaro R. Noninvasive High- Frequency Oscillatory Ventilation: A Retrospective Chart Review. Am J Perinatol. (2020). doi: 10.1055/s-0040-1718738. [Epub ahead of print].

14. Cao H, Li H, Zhu X, Wang L, Yi M, Li C, Chen L, Shi Y. Three noninvasive ventilation strategies for preterm infants with respiratory distress syndrome: a propensity score analysis. Arch Med Sci. (2020) 16:131926. doi: 10.5114/aoms.2020.93541

15. Haidar Shehadeh AM. Non-invasive high flow oscillatory ventilation in comparison with nasal continuous positive pressure ventilation for respiratory distress syndrome, a literature review. J Matern Fetal Neonatal Med. (2021) 34:2900-9. doi: 10.1080/14767058.2019.1671332

16. Mukerji A, Finelli M, Belik J. Nasal high-frequency oscillation for lung carbon dioxide clearance in the newborn. Neonatology. (2013) 103:1615. doi: 10.1159/000345613

17. Owen LS, Morley CJ, Davis PG. Effects of synchronization during SiPAPgenerated nasal intermittent positive pressure ventilation (NIPPV) in preterm infants. Arch Dis Child Fetal Neonatal Ed. (2015) 100:F2430. doi: 10.1136/archdischild-2013-305830

18. De Luca D, Dell'Orto V. Non-invasive high-frequency oscillatory ventilation in neonates: review of physiology, biology, and clinical data. Arch Dis Child Fetal Neonatal Ed. (2016) 101:F56570. doi: 10.1136/archdischild-2016-310664

19. Binmanee A, El Helou S, Shivananda S, Fusch C, Mukerji A. Use of high noninvasive respiratory support pressures in preterm neonates: a single-center experience. J Matern Fetal Neonatal Med. (2017) 30:283843. doi: 10.1080/14767058.2016.1265931

20. Mukerji A, Sarmiento K, Lee B, Hassall K, Shah V. Non-invasive highfrequency ventilation versus bi-phasic continuous positive airway pressure (BP-CPAP) following CPAP failure in infants $<1250 \mathrm{~g}$ : a pilot randomized controlled trial. J Perinatol. (2017) 37:49-53. doi: 10.1038/jp.2016.172

21. Gaertner VD, Waldmann AD, Davis PG, Bassler D, Springer L, Thomson J, et al. Transmission of oscillatory volumes into the preterm lung during noninvasive high-frequency ventilation. Am J Respir Crit Care Med. (2021) 203:998-1005. doi: 10.1164/rccm.202007-2701OC

22. Courtney SE, Asselin JM. High-frequency jet and oscillatory ventilation for neonates: which strategy and when? Respir Care Clin N Am. (2006) 12:453-67. doi: 10.1016/j.rcc.2006.06.005

23. Horbar JD, Carpenter JH, Badger GJ, Kenny MJ, Soll RF, Morrow KA, et al. Mortality and neonatal morbidity among infants 501 to 1500 grams from 2000 to 2009. Pediatrics. (2012) 129:1019-26. doi: 10.1542/peds.20113028 
24. Keszler M, Sant'Anna G. Mechanical ventilation and bronchopulmonary dysplasia. Clin Perinatol. (2015) 42:781-96. doi: 10.1016/j.clp.2015.08.006

25. Kapadia VS, Chalak LF, Sparks JE, Allen JR, Savani RC, Wyckoff MH. Resuscitation of preterm neonates with limited versus high oxygen strategy. Pediatrics. (2013) 132:e1488-96. doi: 10.1542/peds.2013-0978

26. Reyburn B, Li M, Metcalfe DB, Kroll NJ, Alvord J, Wint A, et al. Nasal ventilation alters mesenchymal cell turnover and improves alveolarization in preterm lambs. Am $J$ Respir Crit Care Med. (2008) 178:407-18 doi: 10.1164/rccm.200802359OC

27. Sahni M, Bhandari V. Recent advances in understanding and management of bronchopulmonary dysplasia. F1000Res. (2020) 9:F1000. doi: 10.12688/f1000research.25 338.1

28. Li Y, Wei Q, Zhao D, Mo Y, Yao L, Li L, et al. Non-invasive high-frequency oscillatory ventilation in preterm infants after extubation: a randomized, controlled trial. J Int Med Res. (2021) 49:300060520984915. doi: 10.1177/0300060520984915
Conflict of Interest: The authors declare that the research was conducted in the absence of any commercial or financial relationships that could be construed as a potential conflict of interest.

Publisher's Note: All claims expressed in this article are solely those of the authors and do not necessarily represent those of their affiliated organizations, or those of the publisher, the editors and the reviewers. Any product that may be evaluated in this article, or claim that may be made by its manufacturer, is not guaranteed or endorsed by the publisher.

Copyright $\odot 2022$ Lai, Xie, Chen, Chen, Cai, Wu, Lin and Zheng. This is an openaccess article distributed under the terms of the Creative Commons Attribution License (CC BY). The use, distribution or reproduction in other forums is permitted, provided the original author(s) and the copyright owner(s) are credited and that the original publication in this journal is cited, in accordance with accepted academic practice. No use, distribution or reproduction is permitted which does not comply with these terms. 